\begin{tabular}{|l|c|c|c|}
\hline $\begin{array}{l}\text { ACTA CLASSICA } \\
\text { UNIV. SCIENT. DEBRECEN. }\end{array}$ & LII. & 2016. & \\
\hline
\end{tabular}

\title{
EPIGRAFÍA PÚBLICA Y DEFIXIONES: PARADIGMAS (Y PARADOJAS) DEL OCCIDENTE LATINO ${ }^{1}$
}

\author{
POR CELIA SÁNCHEZ NATALÍAS
}

\begin{abstract}
This paper falls into two main sections. The first deals with the defixio and its traditional definition as an example of so-called private inscriptions. Unlike public epigraphs, which were monumental, crafted by professionals, intended for display, and had (mainly) a commemorative function, defixiones (whether written by magoi or amateurs) are usually considered to be among Antiquity's most private texts. Nevertheless, curse tablets and public inscriptions share a very important feature: both contained messages meant to endure. This specific feature brings us to the second section of this article, which discusses the influence of public inscriptions on curse tablets: to what extent are defixiones a reflection of monumental epigraphy? Aspects such as the ordinatio of the text, the media employed or the way they were displayed (even inside a tomb) are analyzed in this regard. In an attempt to answer these questions, three publicly displayed curse tablets are discussed in depth.

Keywords: Latin defixiones, curse tablets, public inscriptions, PGM.
\end{abstract}

To Professor György Németh, dear colleague, magos and friend with my best wishes for his $60^{\text {th }}$ birthday.

1. Defixiones, ¿documentos privados?

Tal y como sostiene Francisco Beltrán, tradicionalmente, la diferencia entre ámbitos público y privado traza, sin duda, la línea divisoria esencial a la hora

\footnotetext{
${ }^{1}$ Este artículo ha sido redactado durante el disfrute de la "Ayuda para la Especialización de Personal Investigador del Vicerrectorado de Investigación de la UPV/EHU” (2015-2016), en la Universidad del País Vasco. Además, se inscribe en el proyecto de investigación "Procesos de aculturación religiosa en el Mundo Antiguo y en la América colonial" (con referencia HAR 2014-57067-P), dirigido por el Dr. Francisco Marco Simón, a quien agradezco sus comentarios al respecto. Una versión reducida y preliminar de esta investigación se presentó en forma de póster en el XIV Congressus Internationalis Epigraphiae Graecae et Latinae (Berlín, 27-31 de agosto de 2012). En este trabajo, se utilizan las siguientes abreviaturas: $D T=$ Audollent 1904; DTM = Blänsdorf 2012; $P G M=$ Preisendanz 1973²; RIB = Collingwood 1965 y Tab. Sul.= Tomlin 1988.
} 
de realizar una taxonomía de las inscripciones ${ }^{2}$. Con este criterio, se entienden por públicas aquellas piezas de buena factura (realizadas por un lapicida profesional) destinadas a su exposición, que tienen - por lo general - una función conmemorativa. Por su parte, y siguiendo al autor, las inscripciones privadas contienen textos efímeros, redactados por particulares, destinados a una audiencia reducida y que se han conservado - principalmente - porque fueron inscritos en soportes no perecederos.

Por norma general, las defixiones, redactadas normalmente sobre tablillas de plomo $^{3}$, son consideradas una de las tipologías más privadas de la epigrafía clásica $^{4}$, siendo varios los factores que contribuyen a esta categorización. En primer lugar, hay que tener en cuenta que esta era una práctica ilegal: de hecho, sabemos con seguridad que fue sancionada al menos desde la ley de las XII Tablas (donde ya se castigaba a qui malum carmen incantassit ${ }^{5}$ ), de ahí que ni defigentes ni magoi se atreviesen a darle ningún tipo de publicidad. Por otra parte, en favor de esta privacidad están también las características intrínsecas de los propios textos (cuyo contenido era tan secreto como exclusivos los 'lectores' a los que estaban dirigidas), así como los lugares donde las tabellae debían ser depositadas para su definitiva activación, espacios - por lo general recónditos (como tumbas, pozos, santuarios, etc.), y por tanto ocultos al público.

Hasta aquí, es posible afirmar que las defixiones se ajustan a la categoría de inscripciones privadas. Sin embargo, a mi juicio, es necesario matizar un aspecto fundamental, relacionado con la «caducidad» de sus textos. Y es que, si bien es cierto que las inscripciones privadas se caracterizan por contener mensajes por lo general - efímeros, en el caso de las defixiones estamos ante todo lo contrario: al igual que sucedía con la epigrafía pública, los textos de maldición estaban destinados a perdurar en el tiempo ${ }^{6}$. Tanto es así que, objetivo principal de la redacción de la defixio era crear un lazo indisoluble entre el maleficio y la víctima, que se conseguiría mediante la mera inscripción del nombre de la misma ${ }^{7}$. En esta misma línea debe interpretarse el hecho de que las tabellae

\footnotetext{
${ }^{2}$ Beltrán, 2015a, 89.

${ }^{3}$ Aunque no siempre: al respecto, vid. Sánchez Natalías 2011.

${ }^{4}$ Vid. Beltrán 2015a, 90 y 105.

${ }_{6}^{5}$ Lex Duodecim Tabularum, VIII A.

${ }^{6}$ Vid. Piccaluga 2010, 13-14 y 16.

${ }^{7}$ Dentro de la lógica de la magia antigua, la escritura se considera una técnica nociva para la víctima del conjuro, tal y como se deduce a partir de algunas fórmulas recogidas en las propias tabellae (al respecto, vid. Faraone - Kropp, 2010, y, en general, Poccetti 2002, 15). Además hay que tener en cuenta que, en época clásica, el nombre era considerado parte consustancial del individuo y con su inscripción sobre plomo, las cualidades de este último (frío e inerte, y del color de un cadáver, según Aristóteles - apud Plin., Nat. XI 114, 275 -), se transmitirían a la
} 
fuesen depositadas en lugares aislados (e inaccesibles, para evitar que el conjuro pudiera «deshacerse»), en ocasiones acompañadas por la recitación de determinadas fórmulas, destinadas, precisamente, a favorecer supreservación ${ }^{8}$. A estas evidencias de ese intento por perpetuar el mensaje hay que añadir algunas fórmulas recogidas en los propios textos de maldición, como aquéllas mediante las cuales se insta a los dioses a que prolonguen el conjuro «(ad) diem mortis» de la víctima ${ }^{9}$ o la expresión «rediviva» ${ }^{10}$, inscrita sobre el texto principal de una defixio de Lydney Park, en un claro tentativo por reanimar el poder del hechizo a perpetuidad.

\section{Paradigmas (y paradojas) del Occidente Latino}

Esta última fórmula, rediviva, forma parte de una conocidísima defixio in fures hallada en torno a 1817 en el templo de la divinidad romano-céltica Nodens (en Lydney Park). La maldición, redactada por Silvanus contra Senicianus (presunto culpable del robo de un anillo), se inscribe en una lámina de plomo de forma rectangular (de dimensiones 7'5 × $6 \mathrm{~cm}$ ), datada en los siglos IV-V d. E. El texto fue redactado en capitales y presenta una ordinatio muy cuidada, en la que la invocación se dispone a modo de encabezamiento, centrada y ocupando las dos primeras líneas de la inscripción. Estas características, que recuerdan a los cánones de la epigrafía monumental, nos llevan a formular la siguiente cuestión: ¿hasta qué punto las inscripciones públicas pudieron servir como modelo para textos aparentemente tan «privados» como son las defixiones?

Dentro del corpus de maldiciones latinas del Occidente Romano, una gran parte de las tabellae parece haber sido redactada por defigentes individuales ${ }^{11}$, por lo que es razonable suponer que, en ocasiones, estos tomaron como prototipo para su inscripción los textos de su entorno más próximo. Como bien es sabido, la ciudad antigua estaba plagada de epígrafes expuestos en todo tipo de

víctima a través del principio del similia similibus (vid. Sánchez Natalías 2011, 82-83). Por ello, aunque algunos defigentes solicitasen la intervención inmediata de la divinidad, estableciendo plazos mágicos concretos (como, por ejemplo, el de "nueve días", atestiguado en cuatro tabellae - al respecto vid. Marco Simón 2010 -), el maleficio estaba llamado a prolongarse hasta que la víctima recibiese su castigo.

${ }^{8}$ Tal y como se prescribe en $P G M$ VII, 453 y ss.

${ }^{9}$ Según una tabella británica de procedencia desconocida (vid. AE 1991, 1167, 11. 6-7). Expresiones análogas aparecen en una pieza de Mainz (vid. DTM 5, cara A, 11. 7-8: quandius vita vixerit), o la hallada en Rattclife-on-Soar (vid. AE 1993, 1087, 11. 3-4: usque diem quo moriatur).

${ }^{10}$ Como reza la primera línea de la defixio de Lydney Park (al respecto, vid. RIB I 306, con la bibliografía precedente).

${ }^{11}$ Del santuario de Sulis Minerva en Bath contamos con un extraordinario dato: de los 130 textos publicados, sólo dos (Tab. Sul. 95 y 96) fueron redactados por el mismo scriptor. 
espacios públicos (foros, santuarios, necrópolis,...), que en ocasiones, sirvieron - consciente o inconscientemente - como canon para los scriptores. Tal y como defendía Richard Gordon para el caso de las defixiones de la Atenas clásica cuyo texto se presenta en forma de lista (emulando las de los desertores que, para su infamia, se exponían públicamente $)^{12}$, otros aspectos de la epigrafía monumental tienen - a mi parecer - su reflejo en las tabellae latinas. Entre ellos, como veremos, están la (inusual y) cuidada ordinatio de algunos textos, y también cuestiones relacionadas con su enclavamiento, exposición y/o soporte.

En cuanto a la ordinatio, al ya comentado caso de Lydney Park habría que sumar otros ejemplares procedentes de Italia y Britania. Entre ellos, cabe destacar una tabella hallada en una tumba cumana ${ }^{13}$ : la pieza, datable en el S. I d. E., fue inscrita sobre una lámina de plomo rectangular y presenta cinco líneas de texto. Este, bien centrado y con las palabras separadas mediante interpunciones (formadas por un punto situado a media caja de escritura), fue redactado en capitales de módulo variable, dando un mayor tamaño al nombre de la víctima. Como bien sabía el scriptor, este recurso, típico de la epigrafía monumental, estaba destinado, a resaltar la parte esencial del texto. Una estrategia similar es la empleada por Docilianus, autor de una tabella de Bath (Aquae Sulis) datable en el S. II d. E. y redactada en capitales ${ }^{14}$. El texto aparece, de nuevo, centrado, y con las cuatro primeras líneas formando un perfecto encabezamiento en el que el comitente invoca a la divinidad, emulando sin duda los cánones de las inscripciones públicas.

Por lo que respecta al enclavamiento de las piezas, y partiendo de la base de que el clavo es el mejor y más conocido emblema de este ritual mágico ${ }^{15}$, cabe señalar que dentro del Occidente Latino las tabellae asociadas a este elemento (atravesadas y/o depositadas junto al mismo) se corresponden aproximadamente con un $12 \%$ del total. Aunque en ocasiones el clavo perfora un determinado antropónimo o expresión del texto (ahondando -más si cabe- en ese sentimiento de ligadura entre víctima y maleficio ${ }^{16}$ ), lo cierto es que también se dan otros

\footnotetext{
${ }^{12}$ Gordon, 1999.

${ }^{13}$ Con referencia DT 196. Del sur de Italia procede asimismo una pieza osca de gran interés, hallada en Roccagloriosa (vid. Murano 2014, defixio num. 13), cuya primera línea de texto ocupa toda su extensión, a modo de enunciado y a continuación sigue un elenco de antropónimos en forma de columna que aparece en la zona central.

${ }^{14}$ Vid. Tab. Sul. 10 y también 9.

${ }^{15}$ En palabras de Cesano $\left(1961^{2}, 1562\right)$ : "il chiodo trapassante la tabella significava che doveva di necessità avvenire ciò che vi era scritto; come dal fautore della defixio il chiodo era confitto nel piombo così il defisso venina indisolubilmente legato".

${ }^{16}$ Sirva como ejemplo la Tab. Sul. 8, que concluye con un elenco de antropónimos reiterado (en diferente orden) en la cara inversa de la pieza, para que así, el clavo que la atraviesa coincida en ambas caras con el nombre de Anniola, una de las víctimas. Otro ejemplo de interés sería el de
} 
casos en los que estos se sitúan en los ángulos de la tabella, como claros elementos de fijación del soporte a una superficie determinada. Esta disposición se observa, por ejemplo, en una pieza de Cerveteri datada en los siglos II-I a. E., que fue hallada en una tumba de la necrópolis de la Banditaccia ${ }^{17}$. El plomo, de forma rectangular, ha perdido una de sus esquinas, pero conserva los orificios de tres clavos en los ángulos restantes. Evidentemente, estos indican que la tabella había sido fijada a un muro, siguiendo el modus operandi de la epigrafía pública, aunque - en este caso - la pared se encontrase en el interior de una tumba, y el lector al que se dirigía el defigens fuese sobrehumano.

Otro ejemplo similar procede de Caerleon, en cuyo anfiteatro fue hallada una defixio in fures datable en los siglos I-II d. E. y redactada sobre una tabula ansata $^{18}$. La pieza fue perforada en sus ansae con claros fines expositivos, objetivo que aquí se completa además con la elección de un soporte típico de la epigrafía común que en este caso se adapta al mundo de la magia, al haber sido realizado en plomo (con las consiguientes valencias que ello suponía) ${ }^{19}$. Esta transferencia de soportes de un ámbito a otro se observa también en otras provincias, como Hispania, de donde proceden dos maldiciones redactadas sobre sendas tabulae cum capitulo de plomo: concretamente, se trata de dos defixiones in fures datables en el S. II d. E. y halladas en una casa de Santiponce (antigua Italica) y en el santuario de Isis en Bolonia (Baelo Claudia). Según los editores de esta última, las ranuras conservadas en el capitulo de la pieza indicarían que esta habría sido expuesta públicamente ${ }^{20}$.

$\mathrm{Si}$, hasta aquí, los ejemplares comentados evidencian ese (a veces, tenue) reflejo de la epigrafía monumental en un género considerado tradicionalmente tan privado como es el de las defixiones; esta tendencia alcanzará su cénit en

Villepouge Chagnon (vid. DT 111-112), en la que, mediante una fórmula del tipo similia similibus, y refiriéndose a las víctimas, maldice: "sic traspecti sin[t]/ quomodi ille" (Tab. II, 11, 45), interpelación que parece seguida de una perforación en el propio plomo.

${ }^{17}$ Sobre la misma, vid. AE 1961, 207. Otras tabellae que presentan orificios en sus ángulos (en un intento de "exposición"), son las piezas republicanas hispanas procedentes de Carmona (AE 1993, 1008) y El Portal (vid. González Fernández 2015).

${ }^{18}$ Vid. RIB I 323.

${ }^{19}$ Otras defixiones redactadas sobre tabulae ansatae son las halladas en Bodengraven, Italia (loc. inc.), Rottweil, Villepouge Chagnon y quizá Bath (vid., respectivamente, $A E$ 2007, 1029, CIL XI, 6722, 17, Nuber 1984, DT 111-112 y Tab. Sul. 15). Del Norte de África procede asimismo otro texto redactado sobre una tabula ansata, perteneciente a la categoría de los amuletos, según Németh 2012 y también Németh 2013, 73-77 y 235-238.

${ }^{20}$ Vid. Bonneville et al. 1988; para las piezas hispanas, vid. AE 2010, 108. Otro caso de gran interés sería el del plomo galo de Chamalières, redactada sobre una tabella cum capitulo de plomo y descubierta en el santuario galo-romano ubicado la Source des Roches (al respecto vid. Lambert 2002, L-100). 
tres textos que, inequívocamente, y pese a su naturaleza execratoria, forman parte de las inscripciones públicas ${ }^{21}$.

El primero de ellos, y quizá también el más controvertido al carecer de su contexto arqueológico original, proviene de Mérida, más concretamente del pantano de la Albuera (en cuya presa se localizó encastrado hacia 1826). La pieza, datable en el S. II d. E., fue redactada sobre una placa de mármol blanco (de dimensiones $31^{\prime} 5 \times 29 \times 4{ }^{\prime} 5 \mathrm{~cm}$ ), está rota en su parte inferior y en su ángulo superior derecho (vid. figura 1). El texto, enmarcado por una moldura de $6 \mathrm{~cm}$, pertenece a la categoría de las defixiones in fures y dice así: Dea Ataecina Turi-/ brig(ensis) Proserpina/ per tuam maiestatem/ te rogo oro obsecro/ uti vindices quot mihi/ furti factum est quisquis/ mihi imudavit (i) involavit/ minusve fecit [e]a[s res] q(uae) i(nfra) s(criptae) s(unt)/ tunicas VI [...] [pa]enula/ lintea II in[dus] ium cu-/ ius I+C+U[-c.3-]M ignoro IA[---]ius/ VI[---22.

El segundo ejemplar a tener en cuenta es una imprecación pompeyana (vid. figura 2), que en la actualidad forma parte del monumento funerario 23 OS de la necrópolis de Porta Nocera, una tumba 'a edicola' de 6'5 m de altura, donde se encuentra in situ. La imprecación, emplazada bajo la lápida sepulcral, sobresale netamente de la fachada de la tumba, y tiene por protagonistas a dos de sus ocupantes: el defigens (y constructor de la sepultura), que sería el rico liberto Publio Vesonio Filerote y su antiguo amicus (víctima de la defixio), Fausto Orfellio. El epígrafe, datable en el S. I d. E., fue redactado sobre una placa de pizarra de color oscuro (- ¿emulando, quizá, el aspecto del plomo? - de dimensiones $38^{\prime} 8 \times 80^{\prime} 3 \mathrm{~cm}$ ) y reza: Hospes $\cdot$ paullisper $\cdot$ morare $/ \mathrm{si} \cdot$ non $\cdot$ est $\cdot$ molestum $\cdot$ et $\cdot$ quid $\cdot$ evites/ congnosce $\cdot$ ami-cum $\cdot$ hunc $\cdot$ quem $\cdot$ speraveram $\cdot$ mi - esse $\cdot$ ab eo $\cdot$ mihi $\cdot$ accusato/ res $\cdot$ sub-iecti $\cdot$ et $\cdot$ iudicia $\cdot$ instaurata $\cdot$ deis $\cdot /$ gratias $\cdot$ ago $\cdot$ et $\cdot$ meae $\cdot$ innocentiae $\cdot$ omni $\cdot /$ molestia $\cdot$ liberatus $\cdot$ sum $\cdot$ qui $\cdot$ nostrum $\cdot$ mentitur $\cdot /$ eum $\cdot$ nec $\cdot$ di $\cdot$ penates $\cdot$ nec $\cdot$ inferi $\cdot$ recipiant ${ }^{23}$.

Tras su redacción, la defixio fue encastrada en la fachada del monumento y enclavada con cinco pernos de hierro. De ellos, cuatro se sitúan en los ángulos de la pieza (sujetando el epígrafe), mientras que el quinto ocupa una posición preeminente en el centro de la misma. Tal y como ya notara Maria Elefante $(1985,433)$, la clave de lectura del texto reside en este último clavo, cuya situación entre los términos meae e innocentiae, no tendría un valor funcional sino

\footnotetext{
${ }^{21}$ Una cuarta pieza podría ser la inscripción rupestre de Remeseiros, descubierta en 1729 y ya incluida por Hübner en el CIL (II 2476), donde la describía como 'imprecationis vivae rupi inscriptae, similis eius quam supra dedimus inter Emeritenses ad deam Ataecinam directae'.

${ }^{22}$ Vid. CIL II 462= DT 122= AE 2010, 108 (con la bibliografía precedente). Para el teónimo Ataecina, vid. Marco Simón 2011.

${ }^{23}$ Según lección de Elefante, 1985, 434. Una buena síntesis de la historia del monumento (con su correspondiente bibliografía) se encuentra en Williams, 2012, 260-266.
} 
mágico, al intentar fijar de este modo el significado de las propias palabras, subrayando así la inocencia del defigens y condenando a su antiguo amicus a convertirse en un insepulto.

El tercer y último caso procede de Roma: hallado en circunstancias poco claras hacia el S. XVI, el epígrafe se localiza en un altar funerario de mármol (de dimensiones $99 \times 63 \times 51 \mathrm{~cm}$, vid. figura 3) ricamente decorado y datable en el S. I d. E. ${ }^{24}$. El monumento contiene dos inscripciones: la primera de ellas, situada en la base de la cara principal es un epitafio dedicado a la difunta Junia Procula (una niña de casi 9 años de edad), por sus padres, Euphrosynus y -e. El nombre materno, del que se conserva únicamente la última letra, sufrió una damnatio memoriae por las razones expuestas en la segunda inscripción del altar, situada en su cara posterior (la única exenta de decoración vid. figura 4). Se trata de una maldición redactada en versos yámbicos, cuyo texto dice: Hic stigmata aeterna Acte libertae scripta sunt vene/nariae et perfidae dolosae duri pectoris clav $\square u \square m$ et restem / sparteam ut sibi collum alliget et picem candentem / pectus malum com $\square b \square$ urat suum manumissa grati $(i)$ s / secuta adulterum patronum circumscripsit et / ministros ancillam et puerum lecto iacenti / patrono abduxit ut animo desponderet solus / relictus spoliatus senex e(t) Hymno $\{f\} \square e \square$ ade(m) sti(g)m(a)ta / secutis / Zosimum ${ }^{25}$.

Aunque no se menciona explícitamente en el texto, el autor del mismo es con toda probabilidad - el propio Euphrosynus, esposo de la liberta Acte y madre de Junia Procula, quien - tras la muerte de la pequeña - decidió abandonar el hogar familiar en compañía de su amante, llevándose consigo dos esclavos. La ira de Euphrosynus se plasmó en la redacción de este epígrafe ${ }^{26}$, ubicado en el monumento funerario de su propia hija, a la que convirtió - aprovechando su calidad de aoros, como ya notara Judith Evans $(2002,242)$ - en vengadora de la afrenta sufrida.

Estas tres últimas imprecaciones, de cuidada ordinatio y buena factura, fueron redactadas sobre soportes propios de la epigrafía monumental, lo cual las convierte en tres excepciones de notable interés dentro de su categoría. Por norma general, el plomo constituye el soporte fundamental para este tipo de

${ }^{24}$ Este pasó a formar parte de las colecciones romanas de arte clásico de la Vigna of Giovanni Poggi, Villa Giulia y Villa Medici sucesivamente. En la actualidad se conserva en la Galleria degli Uffizi (con número de inventario 950). Vid. Tedeschi y Solin 2011, 265-268.

${ }^{25}$ Según lección de CIL VI 20905; al respecto, vid. Evans 2002.

${ }^{26}$ Resulta interesante la expresión "clavom et restem sparteam" (11. 3-4): como señala Evans (2002, 235-236) esta se localiza en otro epígrafe de Roma (con referencia CIL VI 12649). Aunque cuenta con interesantes paralelos literarios en Plauto y Marcial (vid. Evans 2002, nota 21), en ellos se menciona exclusivamente la soga, y no el clavo. Sin embargo, ¿podría estar en directa relación con el acto en si mismo de defixio, documentado con seguridad en el texto que aquí nos ocupa? 
textos, tal y como lo demuestran los numerosos testimonios literarios y - sobre todo - arqueológicos. No obstante, y si bien es cierto que dentro de los Papyri Graecae Magicae existen numerosas y muy variadas recetas de imprecaciones en las que se contempla el empleo de otros materiales como soporte inscriptorio $^{27}$, también lo es que no existe ninguna prescripción en la que se establezca la utilización de «materiales nobles» para la redacción de una defixio. Su empleo aquí, por tanto, debería ser entendido como un claro reflejo de la epigrafía monumental, en auge en el momento de redacción de estos textos (todos ellos datables entre los SS. I-II d. E.), si tenemos en cuenta el llamado «Epigraphic Habit» $^{28}$.

Así las cosas, y a sabiendas de que la relación entre el soporte y el conjuro está siempre destinada a favorecer el éxito de la praxis mágica, el empleo de estos soportes respondería a la voluntad de los defigentes de exponer públicamente las maldiciones, para mayor infamia de sus adversarios. Exposición, que, por otra parte, implicaba desventajas tanto económicas (debido al sobrecoste de su producción), como penales (dada la ilegalidad de este tipo de prácticas). Sin embargo, y probablemente sabedores de este peligro, los defigentes intentaron sortearlo mediante mecanismos como el anonimato (muy posible en el caso emeritense) o el "camuflaje" de la execración, disfrazada de carmen en Roma, de titutus sepulcralis en Pompei (donde incluso el texto se inicia con la típica fórmula de la epigrafía funeraria Hospes paulisper morare si non est molestum...) y de inscripción votiva en Emerita Augusta.

\section{Bibliografía}

Audollent 1904 = Audollent, A.: Defixionum Tabellae. Quotquot innotuerunt tam in graecis orientis quem in totius occidentis partibus praeter atticas. París.

Beltrán Lloris 2015a = Beltrán Lloris, F.: Latin Epigraphy: the Main Types of Inscriptions. In: Bruun, C. - Edmondson, J. (eds.): The Oxford Handbook of Roman Inscriptions. Oxford, 89-108.

Beltrán Lloris $2015 b=$ Beltrán Lloris, F.: The Epigraphic Habit in the Roman World. In: Bruun, C. - Edmondson, J. (eds.): The Oxford Handbook of Roman Inscriptions, 131-148.

Blänsdorf 2012 = Blänsdorf, J.: Die defixionum tabellae des Mainzer Isis- und Mater-MagnaHeiligtums. Defixionum Tabellae Mogontiacenses (DTM). Mainzer Archäologische Schriften 9. Maguncia.

Bonneville et al. 1988 = Bonneville, J. N. - Dardaine, S. - Le Roux, P.: Belo V. L'Épigraphie: les inscriptions romaines de Baelo Claudia. Publications de la Casa de Velázquez: sér. Archéologie 10. Madrid.

\footnotetext{
${ }^{27}$ Sobre los diferentes materiales empleados como soportes para las defixiones, vid. Sánchez Natalías 2011.

${ }^{28} \mathrm{Al}$ respecto, vid. Beltrán Lloris 2015 b.
} 
Cesano 1961² = Cesano, L.: Defixio. In: Ruggiero, E. de, (ed.): Dizionario Epigrafico di Antichità Romane II-II Roma, 1558-1591.

Collingwood 1965 = Collingwood, R. G. - Wright, R. P. (eds.): Roman Inscriptions of Britain: I, Inscriptions on Stone. Oxford.

Elefante 1985 = Elefante, M.: Un caso di defixio nella necropoli pompeiana di Porta Nocera? PP $225,431-443$.

Evans 2002 = Evans Grubbs, J.: Stigmata aeterna: A Husband's Curse. In: Miller, J. F. - Damon, C. - Myers, K. S. (eds.): Vertis in Usum. Studies in Honour of Edward Courtney. Leipzig, 230-242.

Faraone - Kropp 2010 = Faraone, C. A. - Kropp, A.: Inversion, Adversion and Perversion as Strategies in Latin Curse-tablet. In: Gordon, R. - Marco Simón, F. (eds.): Magical Practice in the Latin West. Papers from the International Conference held at the University of Zaragoza 30 Sept-1 Oct 2005. Religions in the Graeco-Roman World 168. Leiden 381-398.

González Fernández 2015 = González Fernández, J.: Tabella defixionis del siglo I a. C. encontrada en El Portal (provincia de Cádiz). Epigraphica 77, 1-2, 103-116.

Gordon 1999 = Gordon, R. L.: What's in a list? Listing in Greek and Graeco-Roman Malign Magical Texts. In: Jordan, D. R. - Montgomery, H. - Thomasson, E. (eds.): The World of Ancient Magic (Papers from the first International Samson Eitrem Seminar at the Norwegian Institute at Athens. 4-8 May 1997). Bergen, 239-277.

Lambert 2002 = Lambert, P.-Y.: Recueil des inscriptions Gauloises (R.I.G.) Vol. II, Fascicule 2. Textes gallo-romains sur instrumentum, CNRS. París.

Marco Simón 2010 = Marco Simón, F.: Ante dies novem: sobre un cómputo temporal mencionado en algunos textos mágico-religiosos. Palaeohispanica 10, 579-590.

— 2011 = Marco Simón, F.: Duagena, Ataecina: dos divinidades mencionadas en contextos mágicos del Occidente hispano. Mene 11, 45-58.

Murano 2014 = Murano, F.: Le tabellae defixionum osche . Ricerche sulle Lingue di Frammentaria Attestazione 8. Florencia.

Németh 2012 = Németh, Gy.: Helioros. SEBarc 10, 423-435.

- 2013 = Németh, Gy.: Supplementum Audollentianum. Zaragoza-Debrecen-Budapest.

Nuber 1984 = Nuber, H. U.: Eine Zaubertafel aus Schramberg-Waldmössinge, Kreis Rottweil. Fundberichte aus Baden-Württemberg 9, 377-384.

Piccaluga 2010 = Piccaluga, G.: Tecnica grafica e liturgia magica nelle tabellae defixionum. SMSR 76-1, 13-20.

Poccetti 2002 = Poccetti, P.: Manipolazione della realtà e manipolazione della lingua: alcuni aspetti dei testi magici dell'Antichità. In: Morressi, R. (ed.): Linguaggio-linguaggi. Invenzione-scoperta. Atti del Convegno (Macerata-Fermi, 22-23 ottobre 1999). Roma 11-59.

Preisendanz 1973² = Preisendanz, K.: Papyri Graecae Magicae. Stuttgart.

Sánchez Natalías 2011 = Sánchez Natalías, C: Escribiendo una defixio: los textos de maldición a través de sus soportes. $A C D$ 47, 79-93.

Tedeschi y Solin 2011= Tedeschi Grisanti, G. - Solin, H. (eds.) Dis Manibus, pili, epitaffi et altre cose antiche di Giovannantonio Dosio. Pisa.

Tomlin $1988=$ Tomlin, R. S. O.: The Curse Tablets. In: Cunliffe, B. (ed.): The Temple of Sulis Minerva at Bath. Volume 2: the Finds from the Sacred Spring. Oxford, 59-269.

Williams 2012 = Williams, C. A.: Reading Roman Friendship. Cambridge-New York.

(ISSN $0418-453 X)$ 